\title{
Visualization Tools for Vorticity Transport Analysis in Incompressible Flow
}

\author{
Filip Sadlo, Ronald Peikert, Member, IEEE, and Mirjam Sick
}

\begin{abstract}
Vortices are undesirable in many applications while indispensable in others. It is therefore of common interest to understand their mechanisms of creation. This paper aims at analyzing the transport of vorticity inside incompressible flow. The analysis is based on the vorticity equation and is performed along pathlines which are typically started in upstream direction from vortex regions. Different methods for the quantitative and explorative analysis of vorticity transport are presented and applied to CFD simulations of water turbines. Simulation quality is accounted for by including the errors of meshing and convergence into analysis and visualization. The obtained results are discussed and interpretations with respect to engineering questions are given.
\end{abstract}

Index Terms-Flow visualization, vorticity transport, unsteady flow, linked views.

\section{INTRODUCTION}

Flow quality mostly determines the operation of turbomachinery. Especially vortices are known to affect its operation. It is therefore of common interest to understand and to locate the mechanisms of vortex development. The methods to be presented are based on the curl of velocity called vorticity and usually written as $\boldsymbol{\omega}=\nabla \times \mathbf{u}$ when $\mathbf{u}$ is the velocity. Vorticity represents the orientation and angular velocity of local rotation and therefore it is often used as a criterion for the existence of vortices. Depending on the application, the study of vortices can be accomplished by finding high vorticity magnitudes. Silver and Wang [12] identified vortices by connected isosurface components of vorticity magnitude and developed an algorithm for tracking them over time. Strawn et al. [13] computed vortex core lines as height ridges [5] of vorticity magnitude. Also the directional information of the vorticity field has been used in the context of vortex visualization. Banks and Singer [2] base their definition on vorticity and pressure, while Levy et al. [8] define a vortex core as a region where normalized helicity is close to -1 or +1 . Helicity is the scalar product of velocity and vorticity, and normalized helicity is obtained by normalizing both vectors prior to taking the product. It was shown [10] that from this definition of a core region a core line criterion can be derived by locating the points where velocity and vorticity are parallel (or antiparallel) vectors. There are also vortex core detection methods that are not explicitly based on vorticity, in particular the $\lambda_{2}$ method by Jeong and Hussain [6] and the method by Sujudi and Haimes [14]. Both of these methods are based on the velocity gradient and hence make implicit use of vorticity.

On the other hand vorticity is also present in shear flow that does not exhibit swirling motion at all. An example is the Poiseuille flow through a cylindrical pipe. It has a quadratic velocity profile and therefore vorticity magnitude increases linearly towards the wall. In more complex flow fields, such as those of fluid machines, the shear flow can separate from the boundary, this way transporting vorticity into the interior of the flow, and possibly developing into a vortex. Because the vorticity field is divergence-free, it can be visualized by vortex lines (integral curves of vorticity) such that their density is proportional to the magnitude of the field [11]. Laramee et al. [7] use a texture advection method for exploring the relationship between velocity and vortic-

- F. Sadlo and R. Peikert are with the Computer Graphics Laboratory, Computer Science Department, ETH Zurich, Switzerland. E-mail: $\{$ sadlo, peikert $\}$ inf.ethz.ch.

- M. Sick is with VA Tech Hydro, Zurich, Switzerland.

E-mail:mirjam.sick@vatech-hydro.ch.

Manuscript received 31 March 2006; accepted 1 August 2006; posted online 6 November 2006.

For information on obtaining reprints of this article, please send e-mail to: tvcg@computer.org. ity. To our knowledge vorticity has always been visualized as a whole. But we believe that a deeper understanding of the different mechanisms involved in the dynamics of vorticity can be obtained only by visualizing the individual terms of the vorticity (transport) equation.

The main contribution of this paper is a set of tools for the exploration of vorticity distribution and vorticity transport. The latter is described by the vorticity equation containing several terms that can be separated by their physical meaning. Additional terms occur if the viscosity is not uniform e.g. because a two-equation turbulence model is used. For the interactive study of such multimodal data it is beneficial to have multiple linked views available [4], combining techniques from scientific and information visualization.

Section 2 gives some background on vorticity transport and the extension of the vorticity equation to the case of non-uniform viscosity and hence two-equation turbulence models. Methods for the visual analysis of vorticity transport in incompressible flow are developed in Section 3. In Section 4 these methods are applied to industrial CFD simulations in hydromechanical projects and interpretations of the results are given.

\section{VORTICITY TRANSPORT}

In this section two versions of the vorticity equation are described. The first version is applicable to CFD simulations with known uniform viscosity, or in a reduced manner to simulations with unknown viscosity. The second version is applicable to simulations where a spatially varying viscosity is given and known. This is often the case because turbulence models lead to spatially varying (modified) viscosity even for fluids with uniform viscosity.

Scalar quantities which will be used for the analysis are derived for both versions of the vorticity equation.

\subsection{Vorticity Equation for Incompressible Flow}

The flow simulations to be visualized are based on the Navier-Stokes momentum equation for divergence-free flow with uniform density and viscosity:

$$
\frac{\partial \mathbf{u}}{\partial t}+\mathbf{u} \cdot \nabla \mathbf{u}=-\frac{\nabla p}{\rho}+v \nabla^{2} \mathbf{u},
$$

with pressure $p$, density $\rho$, kinematic viscosity $v$, divergence $\nabla \cdot$, and Laplacian $\nabla^{2}$.

Taking the curl of Equation 1 and applying several vector identities leads to the vorticity equation (the reader is referred to e.g. [3] for a full derivation):

$$
\frac{\partial \boldsymbol{\omega}}{\partial t}+\mathbf{u} \cdot \nabla \boldsymbol{\omega}=\boldsymbol{\omega} \cdot \nabla \mathbf{u}+v \nabla^{2} \boldsymbol{\omega}
$$

The first term on the right hand side can be decomposed into vortex stretching and vortex tilting by computing its components parallel and 
perpendicular to vorticity:

$$
\begin{aligned}
\frac{\partial \boldsymbol{\omega}}{\partial t}+\mathbf{u} \cdot \nabla \boldsymbol{\omega}= & \underbrace{(\boldsymbol{\omega} \cdot \nabla \mathbf{u})_{\|_{\omega}}}_{\text {stretching }}+\underbrace{(\boldsymbol{\omega} \cdot \nabla \mathbf{u})_{\perp_{\omega}}}_{\text {tilting }} \\
& +\underbrace{v \nabla^{2} \boldsymbol{\omega}}_{\text {diffusion }} .
\end{aligned}
$$

The stretching term represents the rate of stretching of vorticity. Positive stretching increases vorticity because of conservation of the angular momentum of the fluid element. The tilting term represents the rate of rotation of the vorticity vector and does not change its magnitude. The diffusion term, too, can be decomposed in a component parallel to vorticity and a component orthogonal to it. Again, only the parallel component can change the magnitude of vorticity and therefore the perpendicular component is omitted from the quantitative analysis described in Section 3. This leads to the following equation used for analysis:

$$
\begin{aligned}
\left(\frac{\partial \boldsymbol{\omega}}{\partial t}+\mathbf{u} \cdot \nabla \boldsymbol{\omega}\right)_{\|_{\boldsymbol{\omega}}} & =(\boldsymbol{\omega} \cdot \nabla \mathbf{u})_{\|_{\omega}}+\left(v \nabla^{2} \boldsymbol{\omega}\right)_{\|_{\boldsymbol{\omega}}} \\
& =\sigma+\delta,
\end{aligned}
$$

with stretching $\sigma$ and diffusion $\delta$, both parallel to vorticity. Equation 4 contains third-order derivatives because vorticity is already a derivative. Computing these quantities from CFD results introduces numerical error. In practice, it is usually possible to get first derivatives of velocity directly as additional output from the solver. But even then, second derivatives have to be computed numerically. Theoretically, this can be avoided by computing the diffusion term simply as the difference of the left hand side and the stretching term $\sigma$. However, this would require that Equation 4 really holds for the given data. This is not the case if a turbulence model has been used to compute them. Turbulence models introduce modifications to Equation 1 which obviously lead to modifications in Equation 4. In the following section, the modifications are studied for a special class of turbulence models.

Another reason why Equation 4 cannot be assumed to hold exactly is residual errors in the simulation. From a numerical point of view only moderate simulation accuracy is needed for answering the questions in engineering. Because CFD simulations have a high computational cost, mesh refinement is limited and the simulation is not given the time to fully converge. Additionally, for steady simulations, it is often not possible to achieve a fully-converged solution because the problem would require to be solved time-dependently.

Therefore, unless information on the residuals or on the viscosity is available, only two of the three terms of Equation 4 are available for visualization: The left hand side describes the change of vorticity magnitude for a particle at a given location and time, while $\sigma$ gives the portion of this change due to vortex stretching.

If the available information allows to compute the third term $\delta$, the discrepancy $\Delta$ is computed as the absolute difference between the two sides of Equation 4 and is used as a measure of uncertainty. In this case vortex stretching $\sigma$, vorticity diffusion $\delta$, and discrepancy $\Delta$ are available for visualization.

\subsection{Vorticity Equation with Turbulence Models Included}

Turbulence models were introduced in CFD to obtain good results with a realistic level of spatial discretization (refinement level of the mesh). Two-equation turbulence models (see e.g. [1]) such as the $k-\varepsilon, k-$ $\omega$ and the SST models [9] are based on turbulent viscosity. These models are incorporated into the Navier-Stokes equation by a modified viscosity and a modified pressure:

$$
p^{\prime}=p+\frac{2}{3} \rho k, \quad v_{e}=\frac{\mu+\mu_{t}}{\rho},
$$

where $k$ is the turbulent kinetic energy, $\mu$ is the dynamic viscosity, and $\mu_{t}$ is the turbulent (eddy) viscosity.
The modified Navier-Stokes equation then becomes:

$$
\frac{\partial \mathbf{u}}{\partial t}+\mathbf{u} \cdot \nabla \mathbf{u}=-\frac{\nabla p^{\prime}}{\rho}+v_{e} \nabla^{2} \mathbf{u}+\left(\nabla \mathbf{u}+(\nabla \mathbf{u})^{T}\right) \cdot \nabla v_{e}
$$

The last term of Equation 5 accounts for the fact that turbulent viscosity is in general not uniform, even though the medium is homogeneous. Equation 5 can be simplified using the Jacobian's symmetric part $S$ called strain tensor:

$$
\frac{\partial \mathbf{u}}{\partial t}+\mathbf{u} \cdot \nabla \mathbf{u}=-\frac{\nabla p^{\prime}}{\rho}+v_{e} \nabla^{2} \mathbf{u}+2 S \cdot \nabla v_{e}
$$

Taking the curl of Equation 6, applying the same vector identities as for Equation 3, and applying to the right hand side the additional vector identity $\nabla \times(f \mathbf{v})=f \nabla \times \mathbf{v}+\nabla f \times \mathbf{v}$ leads to the modified vorticity equation:

$$
\begin{aligned}
\frac{\partial \boldsymbol{\omega}}{\partial t}+\mathbf{u} \cdot \nabla \boldsymbol{\omega}= & \underbrace{(\boldsymbol{\omega} \cdot \nabla \mathbf{u})_{\|_{\omega}}}_{\text {stretching }}+\underbrace{(\boldsymbol{\omega} \cdot \nabla \mathbf{u})_{\perp_{\omega}}}_{\text {tilting }} \\
& +\underbrace{v_{e} \nabla^{2} \boldsymbol{\omega}+\nabla v_{e} \times \nabla^{2} \mathbf{u}+\nabla \times\left(2 S \cdot \nabla v_{e}\right)}_{\text {diffusion }}
\end{aligned}
$$

Again, Equation 7 is now restricted to the direction parallel to $\omega$, giving

$$
\begin{aligned}
\left(\frac{\partial \boldsymbol{\omega}}{\partial t}+\mathbf{u} \cdot \nabla \boldsymbol{\omega}\right)_{\|_{\omega}} & =(\boldsymbol{\omega} \cdot \nabla \mathbf{u})_{\|_{\omega}} \\
& +\left(v_{e} \nabla^{2} \boldsymbol{\omega}+\nabla v_{e} \times \nabla^{2} \mathbf{u}+\nabla \times\left(2 S \cdot \nabla v_{e}\right)\right)_{\|_{\omega}} \\
= & \sigma+\delta
\end{aligned}
$$

Having data which solve Equation 5 (up to residual errors), there are now two ways to compute the diffusion, either by computing its subterms as $\delta=\left(v_{e} \nabla^{2} \boldsymbol{\omega}+\nabla v_{e} \times \nabla^{2} \mathbf{u}+\nabla \times\left(2 S \cdot \nabla v_{e}\right)\right)_{\|_{\omega}}$ or by subtracting $\sigma$ from the left hand side as $\delta^{\prime}=(\partial \boldsymbol{\omega} / \partial t+\mathbf{u} \cdot \nabla \boldsymbol{\omega})_{\|_{\omega}}-\sigma$.

The discrepancy $\Delta=\left|\delta-\delta^{\prime}\right|$ can again be taken as a measure for the uncertainty of the obtained result. It is equal to the discrepancy between the two sides of Equation 8 .

Here, in contrast to Section 2.1 without additional information, vortex stretching $\sigma$, vorticity diffusion $\delta$, and the discrepancy $\Delta$ are available for visualization.

\section{Methods for Vorticity Transport Analysis}

The analysis of vorticity transport is based on pathlines because Equation 3 is a convective derivative of vorticity with respect to velocity. This way, a pathline can be used to visualize the vorticity transport effects that a fluid element experiences on its path through the flow. Visualizing differential quantities on pathlines avoids integration along the transport paths and hence error accumulation due to integration errors and numerical discrepancy in the data. Computing pathlines upstream or downstream from points of interest allows the researcher to analyze the flow behavior with respect to that region and time.

The quantities of interest are sampled along the pathline and plotted as described in Section 3.2. Selected quantities are also visualized directly on the pathline as described in Section 3.3 and Section 3.4. Vorticity magnitude is mapped to the thickness of the pathlines together with parallel projection rendering. Vorticity orientation is visualized by adding vorticity streamlets to the pathlines. Where appropriate, wall distance can be visualized using "support columns". The quantities could be computed during line integration. It was chosen to precompute them, in order to achieve short response times of the interactive analysis. All line integrations are performed with fourth-order Runge-Kutta integration. 


\subsection{Quantities}

The quantities selected for the basic analysis as described in Section 2.1 include vorticity magnitude, its rate of change along the pathline, the stretching term $\sigma$ and the distance to the boundary. As already mentioned, the focus of the quantitative analysis is on the magnitude of vorticity, not its orientation. Vortex tilting can not change the magnitude and therefore it is actually omitted in the visualization.

If the extended analysis is possible, the diffusion term $\delta$ and the discrepancy $\Delta$ are used as additional quantities, and derived from them also the relative error

$$
\varepsilon=\frac{\left|\delta-\delta^{\prime}\right|}{|\sigma|+|\delta|} .
$$

If available, the residuals as a further data channel can be included in the visualization. Also the Courant number giving indications on possible convergence problems can be taken for comparative visualization.

Because of Equation 4, $\sigma$ and $\delta$ represent a decomposition of the rate of change of the vorticity magnitude into the two mechanisms of vortex stretching and vorticity diffusion. The visualization methods should take this decomposition property into account.

\subsection{Pathline Plots}

As a first type of visualization, a 2D plot is generated for the pathline in question. The abscissa of the plot represents either time or arc length along the pathline while the quantities are mapped to the ordinate. The purpose of having this basic type of visualization available in our toolkit is to have an undistorted view of the pathline and at the same time being able to see more different quantities than would be possible with a condensed representation.

The quantities $\sigma$ and $\delta$ are plotted relative to the plot of vorticity magnitude in order to meet their decomposition property. If positive, they are plotted as area above the plot of vorticity magnitude, otherwise they are plotted as area below it. Stretching forms the inner band while diffusion forms the outer. Figure 1 shows an example result. The discrepancy $\Delta$ is added as another band below the existing plot. This visualization shows the proportion of the two terms as well as their total effect to vorticity magnitude. Because $\sigma, \delta$, and $\Delta$ have different units than vorticity magnitude, the values of $\sigma, \delta$, and $\Delta$ can be freely scaled by a user-defined factor for obtaining significant plots. The sum of the unscaled $\sigma$ and $\delta$ corresponds to the slope of the vorticity magnitude plot, up to the discrepancy $\Delta$.

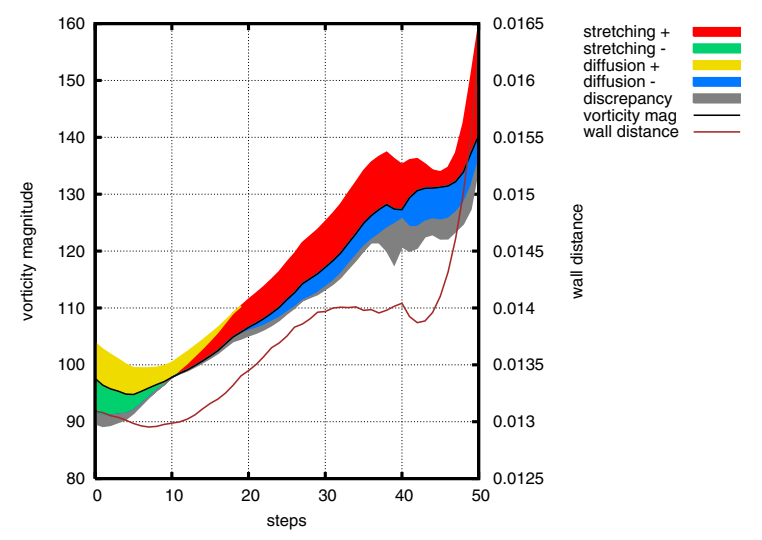

Fig. 1. Plot along a single pathline, corresponding to Figure 2.

\subsection{Striped Pathlines}

Striped pathlines are introduced to show several of the computed quantities at their physical location on sample points along the pathlines. The idea is to provide a condensed visualization on an interactively seeded group of pathlines. Any of the pathlines can then be selected for closer inspection by generating a pathline plot as a linked view.
A tube is generated along each pathline, with radius representing vorticity magnitude, similar to the iconic stream tube [15]. The pathline is divided into segments of constant user-defined length (or time for also visualizing velocity) and the corresponding segments of the tube are used for visualization. The quantities for visualization are sampled at the mid of the pathline segments. Three quantities are visualized by color stripes on the tube segments: Stretching $\sigma$ and diffusion of vorticity $\delta$ as well as the error $\varepsilon$.

Data Stripes: The coloring strategy for the data stripes is chosen according to the pathline plots of Section 3.2. A color pattern proportional to $\sigma$ and $\delta$ can be obtained by looking at a column of the plot area band that represents them. The idea is to map this proportional pattern to the section of the tube using color stripes. Transverse stripes are chosen for better readability. Since stretching comes first (is the inner band) in the plots, it is used for the first stripe and diffusion is used for the second stripe. This coding also indicates flow direction. For normalized visualization of the proportion only, the two-color pattern is scaled to fit the tube segment length. If also the absolute value of stretching and diffusion is to be visualized, a third stripe is used as a filler and colored white. The scaling is determined in this case from $\max (|\sigma|+|\delta|)$ over the pathline or set of pathlines. Because of data peaks along the pathline, this scaling can lead to unsatisfactory visualization and therefore the user is allowed to scale the stripe width. This will lead to clipping at the peaks and allows the user to inspect the region of interest. Clipping is indicated by a black stripe of predefined width. Figure 2 shows an example.
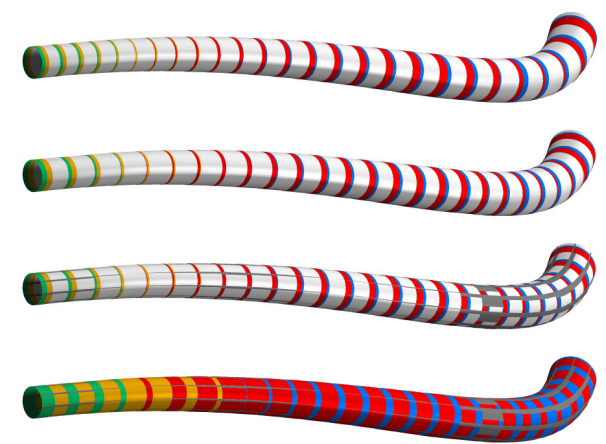

(e)

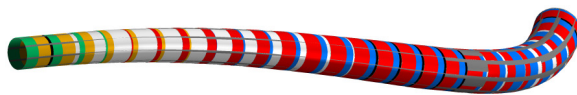

(f)

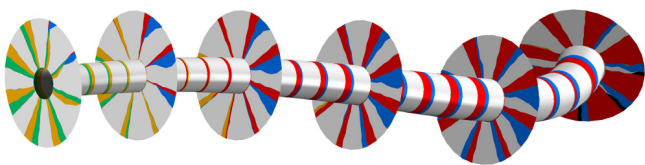

(g)

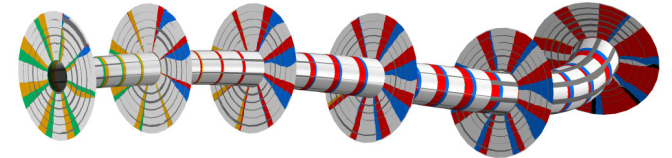

Fig. 2. Striped pathline modes using color scheme of Figure 1. Evenlytimed segment lengths indicating velocity (a), evenly-spaced segment lengths (b), same as (b) but with error stripes (c), with additional normalization of the stripes (d), scaling instead of normalization (e), same as (b) but with additional striped slices (f), and additional error stripes (g). Tube radius represents vorticity magnitude.

Error Stripes: According to the role of error, the error stripes are chosen orthogonal to the data stripes, resulting in longitudinal stripes. The width of the error stripes is proportional to $\varepsilon$ (Equation 9). At full error $(\varepsilon \geq 1)$ the complete tube segment is covered by error stripes leaving no data stripes visible. At zero error no error stripe is visible and all is covered by data stripes. More than one error stripe is generated to avoid its occlusion but the number is kept low to preserve resolution. Figure 2 shows an example. 


\subsection{Complementary Visualization Techniques}

While the two techniques of pathline plots and striped pathlines already provide a multitude of data, these are all local data, and combining them with some contextual visualization is necessary. Since we are concerned mainly with data exploration rather than presentation, complementary visualizations can be easily added or removed during inspection of the data. We describe in the following a few techniques that turned out to play well together with the proposed basic techniques.

Slices: Until now, the visualization is confined to the pathlines. A single pathline visualizes the advective aspects of vorticity well but does not allow to see the local neighborhood of the pathline. However, if there is e.g. gain of vorticity by diffusion on the pathline, one would like to know where it diffuses from. This can be analyzed by generating other pathlines nearby, but only in the case that the pathlines pass the region in question at the same time.

An alternative and more systematic method is to generate perpendicular slices on the pathline, sample the quantities on the slices, and to apply the same color coding as for the striped pathlines. All modes of the pathline stripes are implemented for the slices, too. The circular slices are divided into sectors and this time the data stripes are radial and the error stripes are circumferential. This gives a continuous visualization with increasing radius and contrasts well to the transverse data stripes on the tube. Figure 2 shows an example.

Vorticity Streamlets: So far, only the magnitude of vorticity is visualized. This is sufficient for the quantitative analysis, but it does not produce a complete image. Therefore streamlets of vorticity are added to the pathline visualization. The length of the streamlets is defined by a user-defined time step. This makes them consistent with the tube radius since the tube radius is used to visualize vorticity magnitude. Vorticity streamlets disappearing inside the tube indicate high normalized helicity which by Levy's criterion is associated with swirling flow. Two streamlets are computed at each seed, one in positive and one in negative vorticity direction. Due to the analogy between vorticity and magnetism, it is natural to interpret the streamlets as a kind of compass-needles with possibly bent ends that follow the field. Figure 3 shows an example.

Wall Distance Indicators: Wall distance plays an important role in the analysis of vorticity because of the vorticity present in boundary shear flow. Another reason for keeping an eye on wall distance is that the quality of simulation as well as the quality of the derivatives is often low close to the wall. Wall distance can be visualized e.g. by color coding of the vorticity streamlets. But this adds another type of colored stripes and therefore disturbs the visualization of the pathlines. Therefore "support columns" are introduced. These are tubes that indicate the shortest distance from the curve to the wall. The tubes have a top face that is located at the pathline and follow to the point on the wall with shortest distance to the point on the pathline. They can be computed by streamline integration along the negative gradient of wall distance until the boundary is reached. Without additional information, it would not be possible to tell the length of a tube because of the projection. Therefore the tubes are continuously colored by distance to the boundary. This allows to see the value of wall distance. At the same time the long tubes represent a color legend when seen from the side. If the tube radius is large enough, the top faces of the tubes are well visible when looked from the top, even together with the pathline tube. In this case the color of the top face indicates wall distance. Figure 4 shows an example.

Velocity-Vorticity Streamline Rakes: So far, the described vorticity streamlets are usually chosen shorter than the pathline on which they are generated, because they only add additional information. However, there are applications where the opposite role is valuable. This leads to a special case of streamline rakes, where a single field line is generated interactively and other field lines are started from that curve in evenly-spaced or evenly-timed distribution. Doing this for the same field does not deliver additional information. But velocity and vorticity are particularly suitable because they are sufficiently close to perpendicular in shear flow. The rake with a velocity streamline as primary line and vortex lines starting from it (denoted here as $(u, \omega)$ streamline rakes) can be used for the visualization of boundary shear flow. The rake with a vortex line as primary line and velocity streamlines starting from it (denoted here as $(\omega, u)$ streamline rakes) can be used for visualizing separation of boundary shear flow. Figure 12 shows an example where both types of rakes are used. The described type of rakes has some advantages compared to conventional streamline rakes: The user is freed from the tedious process of rake orientation, a single selection operation already defines the rake. Another advantage is that it allows to generate curved rakes, e.g. following the boundary, also by a single selection operation. Extending the concept of $(u, \omega)$ rakes to time-dependent data does not seem to be usable due to interpretation difficulties. The extension of $(\omega, u)$ rakes to time-dependent data makes sense and could be used as in the steady case of Section 4.4.

\subsection{Methodology}

For the analysis of vortex regions, the pathlines are interactively seeded at vortex cores with high vorticity, on tubes around the cores, or on isosurfaces of vorticity magnitude or $\lambda_{2}$ (at given time in the transient case) and integrated in upstream direction. This allows to analyze the mechanisms that are involved in the development of the given vortex. The vortex cores are either computed according to either Levy et al. [8] or Sujudi and Haimes [14]. Another possibility is to seed the pathlines at regions on the boundary where vorticity is advected away and computing the pathlines in downstream direction. This allows to analyze how and where the vorticity is transported.

Pathlines are often computed in upstream direction in order to analyze the development of the vortex. However, it has to be noted that the pathline visualization has to be interpreted always in downstream direction because $\sigma$ and $\delta$ are computed in positive time. The different modes of Section 3.3 and Section 3.4 are selected interactively until satisfactory visualization is achieved.

Slices, streamlets and wall distance indicators (Section 3.4) are placed either at evenly timed or evenly spaced positions along the pathline. When using wall distance indicators and vorticity streamlets at the same time, it is preferable that the vorticity streamlets get placed at the same position as the wall distance indicators. This improves the perception of streamlet orientation because either both positive and negative parts of the streamlet are visible and hence in the plane of the top face of the tube, or one disappears inside the tube. Another benefit is that the top faces of the support tubes have the same orientation as the wall. This facilitates inspection of vorticity in boundary shear flow because vorticity is parallel to the wall in the general boundary shear flow case.

\section{REsULts}

The methods are applied to different CFD simulations from industry. The first example is the flow inside the distributor ring of a Pelton turbine, which was computed with a symmetry boundary condition. The second example is the free-surface simulation of the basin of a river power plant. Finally, the methods are applied to the simulation of a vortex rope in a draft tube of a Francis turbine. The former two are steady type simulations while the latter is unsteady. Different flow phenomena are examined using the described methods.

\subsection{Separation Vortex (Pelton)}

The first region of inspection in the Pelton dataset is the large separation vortex at the entrance of the first injector. Figure 3 shows a set of pathlines started in upstream direction from the downstream end of its core line. Figure 4 shows a selected pathline from this set and Figure 5 shows the corresponding plot. The images show a part of the distributor ring of a Pelton water turbine. The flow comes from the bottom of the image and follows the distributor ring to the right upper corner. Part of the flow branches off into the injector at the left upper corner of the image.

We start the visual examination at the upstream end of the pathline and follow it downstream. From the plot or from the distance indicators we know that the pathline is close to the wall in that region. There is high vorticity magnitude and this indicates together with the 


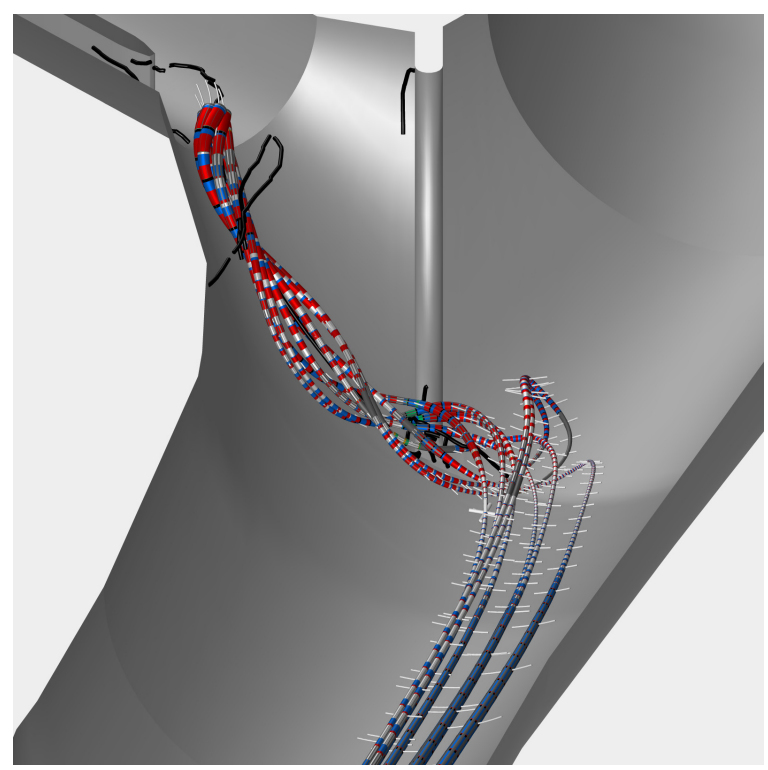

Fig. 3. Separation Vortex. Vorticity transport visualized by a set of striped pathlines, backward integrated from a seed circle at the downstream end of the vortex core line (black). Visualization is augmented by vorticity streamlets (white).

perpendicular orientation of the vorticity streamlets that the pathline is part of the boundary shear flow there. There is large error at the beginning. This can be explained by the fact that simulation accuracy and quality of the computed derivatives are usually low close to the wall. However, it can be seen from the plot, that the error reduces quickly to a practical level as the pathline gains distance to the wall. Vorticity is diffusing from the boundary shear flow in this region.

As next, the pathline starts to deviate into the injector. It can be seen that this is near the upstream end of the separation vortex core line structure, and that the pathline swirls around the core of that vortex for the rest of its way. Here we observe the typical situation that vorticity is mostly gained by stretching in the region of vortices with simultaneous loss by diffusion. The positive stretching of vorticity is often observed in vortices which are located at regions of increasing velocity, e.g. the outlets. The velocity component parallel to vorticity usually increases downstream in these regions, leading to stretching of the fluid and hence its vorticity. The negative diffusion of vorticity is explained by the locally high vorticity magnitude typically found in the core region of a vortex.

\subsection{Recirculation and Vortex (Pelton)}

The entrance of the second injector shows a quite different flow pattern. Instead of a clear separation vortex, a short vortex connected to a large recirculation region is observed. Figure 6 shows a set of pathlines seeded at the downstream end of the vortex, Figure 7 a selected pathline, and Figure 8 the corresponding plot. Again, the flow comes from the lower left corner of the image and part of it is branching off into the injector.

Generally, recirculation zones are often directly related to the boundary layer: They occur where the boundary layer separates from the wall. Again, the upstream parts of the pathlines are very close to the boundary. By observing the almost constant tube width, vorticity transport from the boundary shear flow can be identified.

The upstream parts of the pathlines belong to the recirculation zone, identified by vertical vorticity streamlets. Some of the pathlines follow a loop through the recirculation zone before they reach the vortex. Part of this loop exhibits (vertical) vorticity streamlets which are consistent with the recirculation zone. In the other part of the loop, vorticity orientation is consistent with the vortex. The dominant mechanisms in this example are therefore the transport of vorticity from the boundary

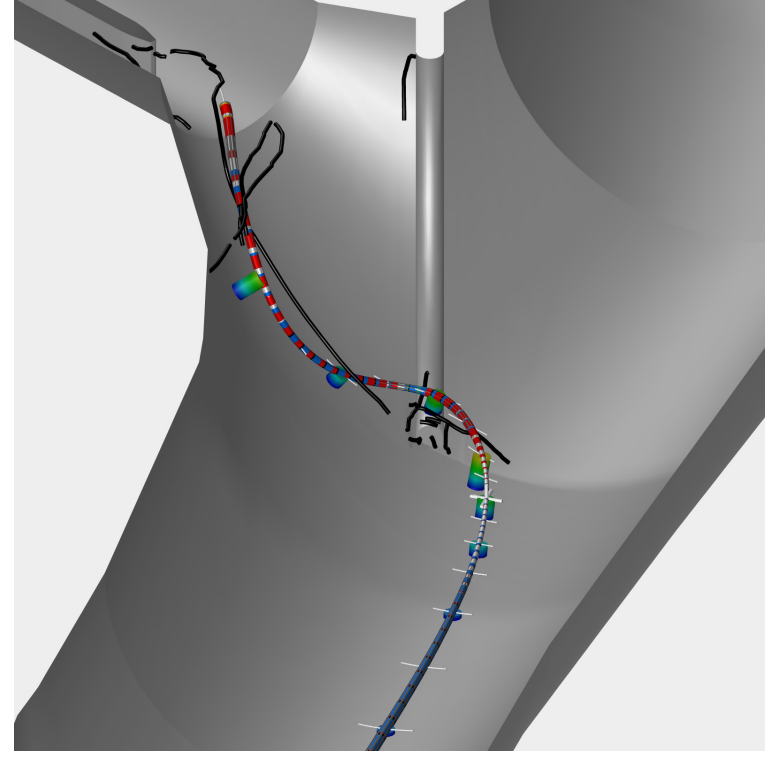

Fig. 4. Selected pathline from Figure 3 with wall distance indicators (colored cylinders).

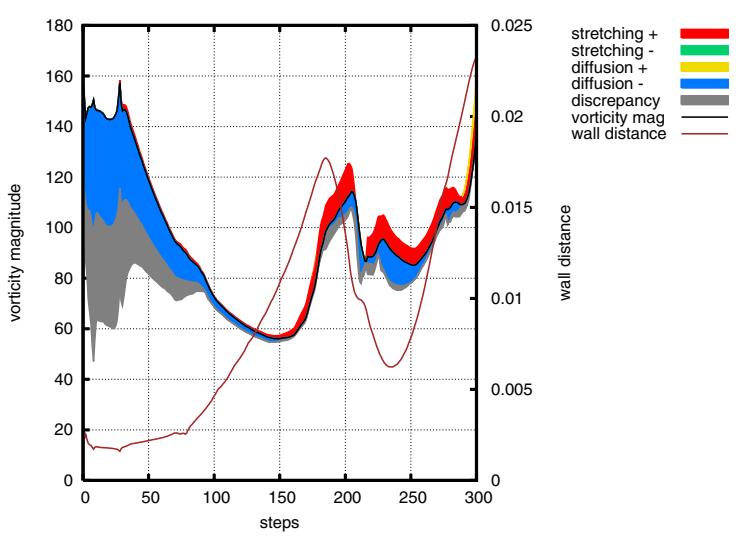

Fig. 5. Pathline plot corresponding to pathline of Figure 4 .

shear, followed by vortex tilting in the recirculation zone and finally vortex stretching plus diffusion in the vortex region.

\subsection{Vortex at Bifurcation (Pelton)}

In this section a vortex near the sickle of the fourth Pelton injector is analyzed. The sickle is a plate at each bifurcation of a Pelton distributor which is inserted for mechanical reasons. Figure 9 shows a set of upstream pathlines, started at the downstream end of the core line. Figure 10 shows the plot corresponding to the selected pathline. Following the pathlines in downstream direction, part of them gets very close to the sickle where vorticity is obtained from the boundary shear flow and then transported into the vortex. Again, vorticity is reinforced by stretching with simultaneous loss by diffusion in the vortex region. Some of the regions where the pathlines are close to the wall show high discrepancy $\Delta$, visible as the gray peak in the plot. The visualization of the Courant number in Figure 11 confirms that there are possible problems with simulation numerics in this region, comprised in the outermost layer of grid cells. This is a case where the computed diffusion is not reliable and hence is an example where the basic method (Section 2.1) could be appropriate. 


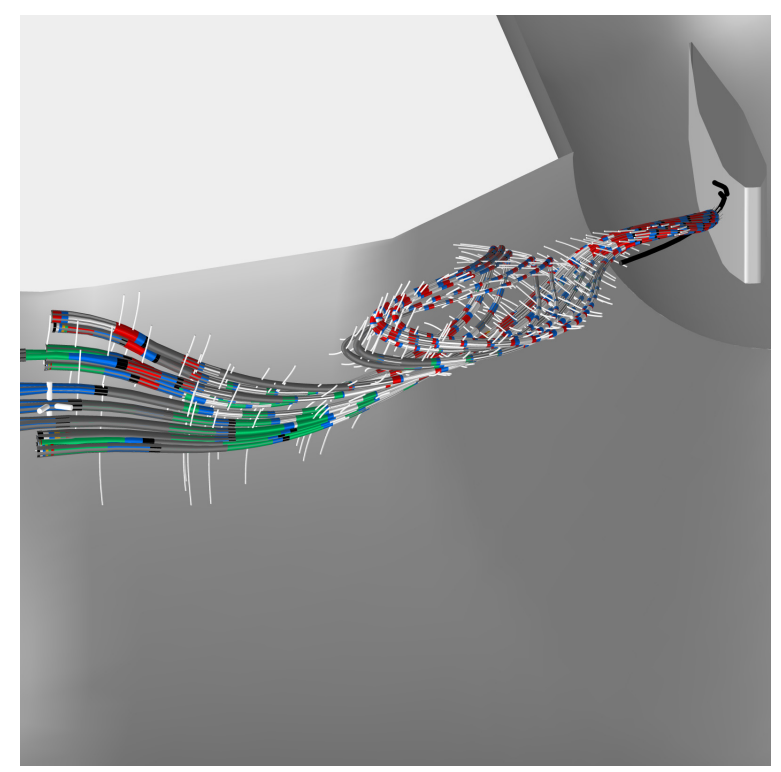

Fig. 6. Recirculation region. Pathlines computed in upstream direction from a circle at downstream end of vortex core line. Vorticity streamlets allow to identify a recirculation zone (left, vertical streamlets) and a vortex region (right, horizontal streamlets).

\subsection{Vorticity Stretching at Boundary Layer Separation (River Power Plant)}

This section examines a region in the river power plant dataset near a free-surface vortex (see Figure 12). The flow comes from the bottom of the image and goes around the housing of the generator, visible at the right side of the image. A streamline is generated interactively close to the bottom of the basin and used for generating a rake of vortex lines, as described in Section 3.4, for the visualization of the boundary shear flow. Some of the vortex lines detach in the region of the vortex and cover it. In order to analyze the flow in the region of detachment, a complementary rake is interactively generated close to the wall inside the vortex region. It can be seen that its primary vortex line keeps well inside the vortex region, due to the orientation of near-wall vorticity inside the vortex region. The complementary rake is built using striped pathlines as shown in Figure 13. This time, evenly-spaced tube segments are used instead of evenly-timed segments.

The main feature in this region is the vorticity gain by vortex stretching after the pathlines have left the boundary shear flow. Stretching is due to the increasing velocity component perpendicular to the wall which is roughly the direction of vorticity, visualized by vorticity streamlets in Figure 13.

\subsection{Transient Vortex Rope (Draft Tube)}

In this section a transient simulation of a vortex rope in the draft tube of a water turbine is examined. The inlet boundary condition was determined empirically from measured data on a slice through an already developed vortex rope. To study the vorticity transport into the vortex, its vortex core line is determined for the last time step of the simulation and used for seeding pathlines in upstream direction as shown in Figure 14. Additionally, the vorticity transport in the boundary layer is visualized by six pathlines seeded on a circle at the inlet and computed in downstream direction.

The pathlines that lead into the vortex exhibit strong alternating gain and loss of vorticity by stretching. It can be seen from Figure 15 that most of the vorticity that is first gained by stretching is lost on the way again by negative stretching, but after all, most of the vorticity at the inlet boundary condition reaches the vortex core line of the last time step. The pathline that is seeded at the lower end of the core line shows the fact that vorticity magnitude is low around the vortex. It can be summarized that the vorticity concentrated in the center region of the

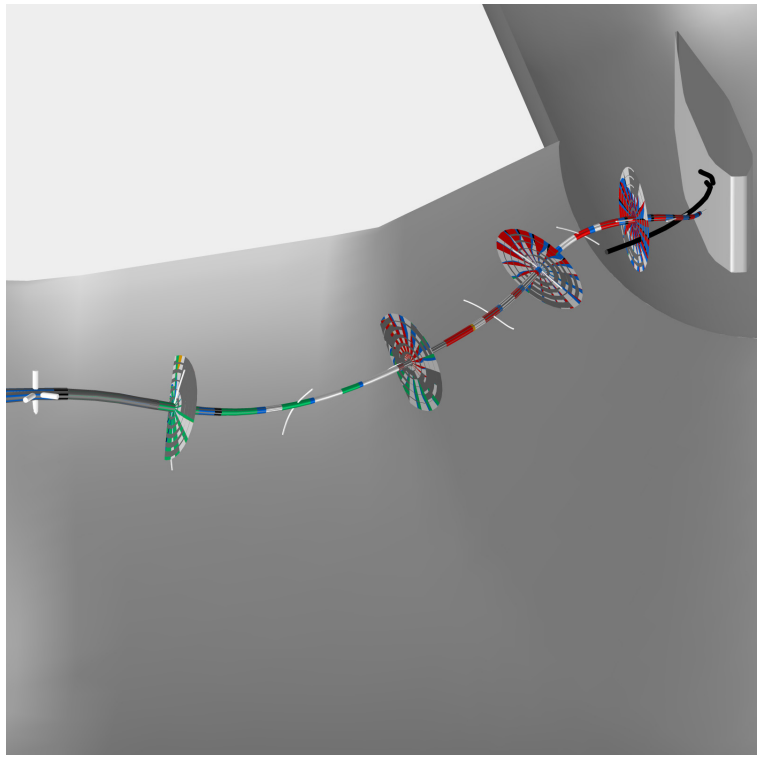

Fig. 7. Selected pathline in Figure 6 with slices showing asymmetrical distribution of the quantities in the neighborhood of the pathline.
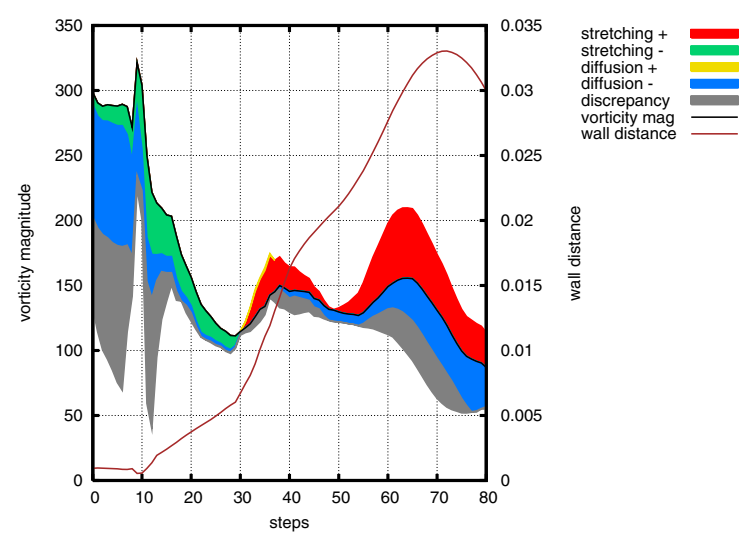

Fig. 8. Pathline plot of selected pathline in Figure 6.

inlet boundary condition, representing the vortex rope, is responsible for the development of the vortex rope.

The six pathlines of the boundary layer first show gain of vorticity by diffusion and later loss of vorticity by diffusion. In between, vorticity is also increased by stretching. This can be identified as development of the boundary shear flow of the draft tube. The asymmetry of the boundary shear flow (the pathline at the back of the tube has larger radius) originates from the helical and hence asymmetric structure of the vortex rope.

\section{Conclusion}

Different methods for the visualization of vorticity transport in unsteady flow have been presented. Integrating various tools based on scientific and information visualization techniques allows both qualitative and quantitative analysis by combining them flexibly in an interactive environment. The visualization toolkit gave deeper insight into the vortex dynamics of the examined cases, revealing how vorticity and vortices are reinforced or weakened by the main flow field. As discussed at the various examples, this method has the potential to support a more thorough understanding of complex flow patterns and might become beneficial in the development and application of turbulence models.

The strength of this method is the fact that a complex flow field can be visualized in a compact way without the need of switching forth and 


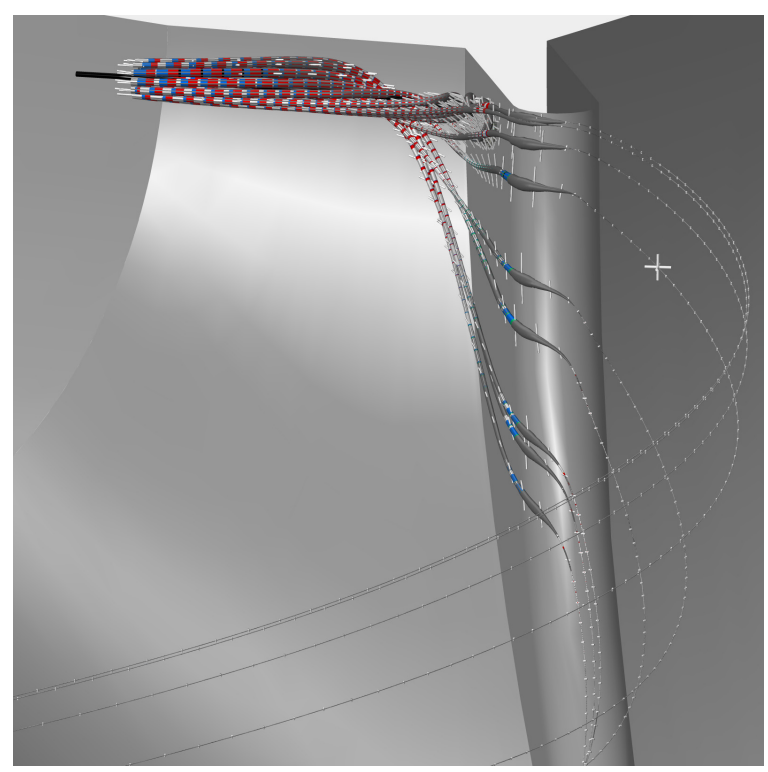

Fig. 9. Set of upstream pathlines, started on a circle at the downstream end of the core line (black). Vorticity increases rapidly as pathlines pass the sickle in downstream direction.

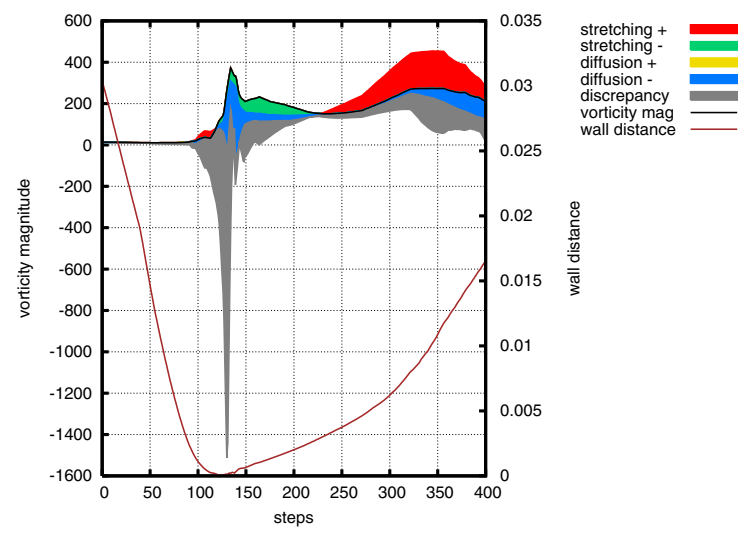

Fig. 10. Cropped pathline plot of sickle vortex corresponding to selected pathline in Figure 9.

back between different representations. As a limitation of the system, one can say that the visualization is quite complex and that it requires some familiarity with the method and the underlying physics.

For future work, we plan to support other turbulence models besides two-equation models, in particular the RSM model to broaden the application range. We expect the problem of high discrepancy to reduce in the future as increasing computing power will allow more accurate and hence more physically correct simulations.

\section{ACKNOWLEDGEMENTS}

The authors would like to thank France Suerich-Gulick (McGill University), Hélène Garcin, Etienne Parkinson and Peter Stein (all VA Tech Hydro) for preparing the data and many fruitful discussions. This work was funded by Swiss Commission for Technology and Innovation grant 7338.2 ESPP-ES.

\section{REFERENCES}

[1] CFX-5 User manual. AEA technology, Harwell, UK, 2002.

[2] D. C. Banks and B. A. Singer. A predictor-corrector technique for visualizing unsteady flow. IEEE Transactions on Visualization and Computer Graphics, 1(2):151-163, 1995.

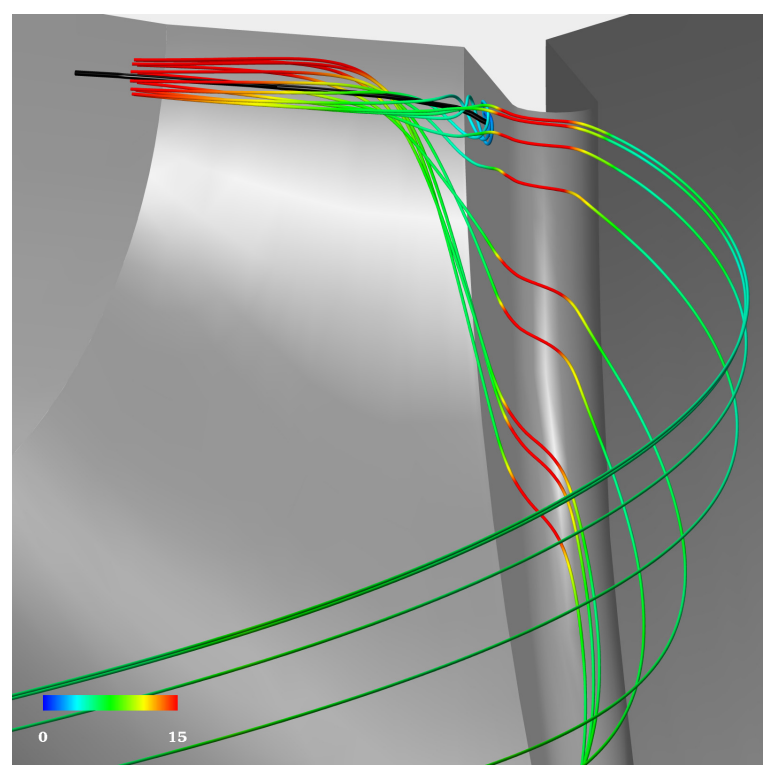

Fig. 11. Visualization of the Courant number. Comparison with Figure 9 shows that the error on the striped pathlines correlates with high Courant number.

[3] G. K. Batchelor. An Introduction to Fluid Mechanics. Cambridge University Press, 1967.

[4] H. Doleisch, M. Gasser, and H. Hauser. Interactive feature specification for focus+context visualization of complex simulation data. In VisSym '03: Symposium on Visualisation, pages 239-248, 2003.

[5] D. Eberly. Ridges in Image and Data Analysis. Computational Imaging and Vision. Kluwer Academic Publishers, 1996.

[6] J. Jeong and F. Hussain. On the identification of a vortex. Journal of Fluid Mechanics, 285:69-94, 1995.

[7] R. S. Laramee, C. Garth, J. Schneider, and H. Hauser. Texture advection on stream surfaces: A novel hybrid visualization applied to cfd simulation results. In EuroVis 2006: Symposium on Visualization, May 2006.

[8] Y. Levy, D. Degani, and A. Seginer. Graphical visualization of vortical flows by means of helicity. AIAA, 28(8):1347-1352, 1990.

[9] F. R. Menter. Zonal two equation k-omega turbulence models for aerodynamic flows. In 24th AIAA Fluid Dynamics Conference, pages 1-21, 1993.

[10] R. Peikert and M. Roth. The parallel vectors operator: a vector field visualization primitive. In Proceedings of IEEE Visualization, pages 263 $270,1999$.

[11] F. Sadlo, R. Peikert, and E. Parkinson. Vorticity based flow analysis and visualization for pelton turbine design optimization. In Proceedings of IEEE Visualization, pages 179-186, 2004.

[12] D. Silver and X. Wang. Volume tracking. In Proceedings of IEEE visualization, pages 157-164, 1996.

[13] R. C. Strawn, D. N. Kenwright, and J. Ahmad. Computer visualization of vortex wake systems. American Helicopter Society 54th Annual Forum, May 1998.

[14] D. Sujudi and R. Haimes. Identification of swirling flow in 3d vector fields. In 12th AIAA Computational Fluid Dynamics Conference, pages 95-1715, 1995.

[15] S.-K. Ueng, C. Sikorski, and K.-L. Ma. Efficient streamline, streamribbon, and streamtube constructions on unstructured grids. IEEE Transactions on Visualization and Computer Graphics, 2(2):100-110, 1996. 


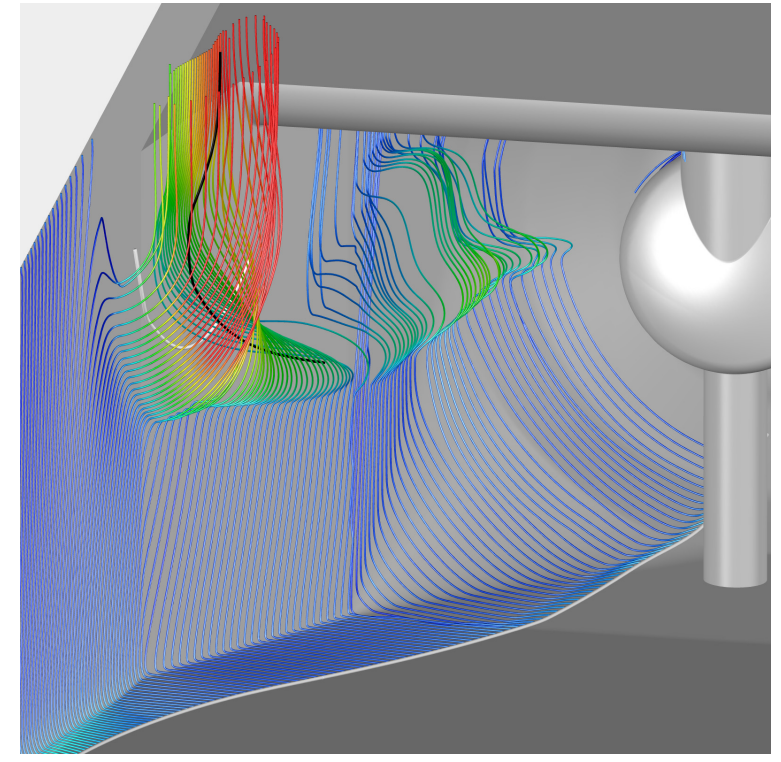

Fig. 12. Separation at large free-surface vortex. Core line of vortex is black. Vortex lines of the boundary shear flow are colored by distance to the boundary. The vortex lines of the boundary shear flow detach at the vortex and cover it. A vortex line has been generated close to the wall inside the vortex region (white tube).

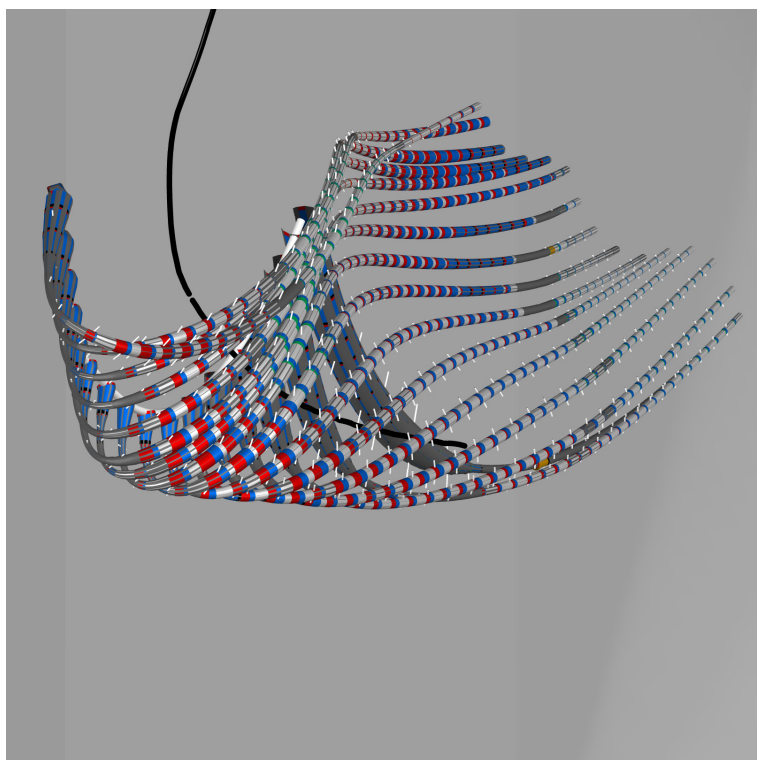

Fig. 13. Close-up view of vortex region in Figure 12 showing a rake of striped pathlines computed in downstream direction, started from the vortex line (white tube) of Figure 12.

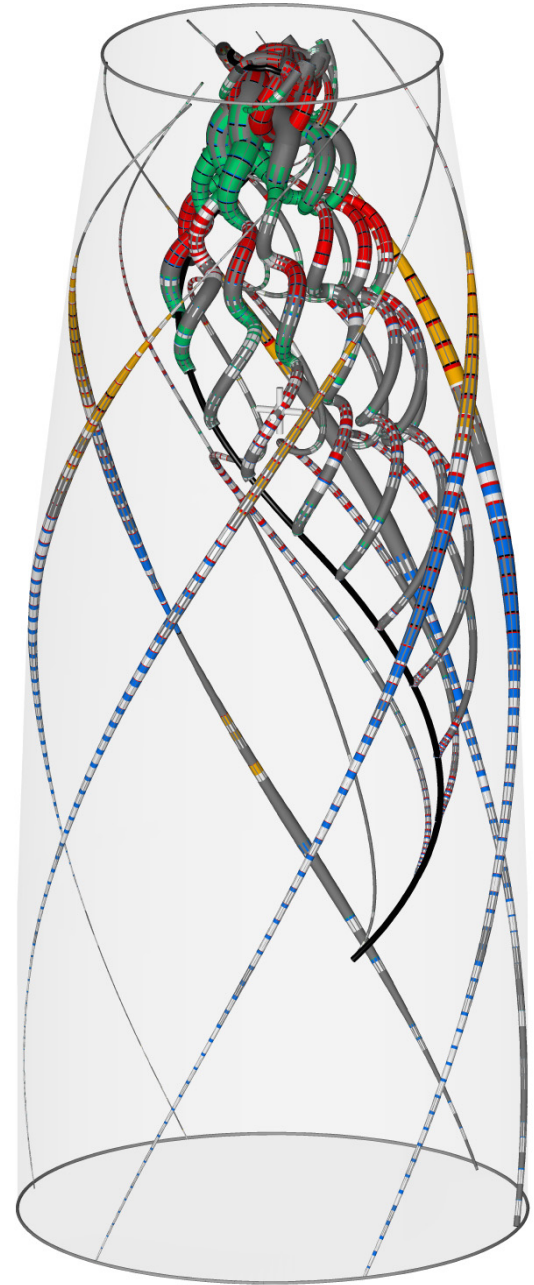

Fig. 14. Transient vortex rope simulation. Pathlines computed in upstream direction from vortex core line of last time step (black tube). Six pathlines computed in downstream direction visualize vorticity transport in the boundary shear flow.

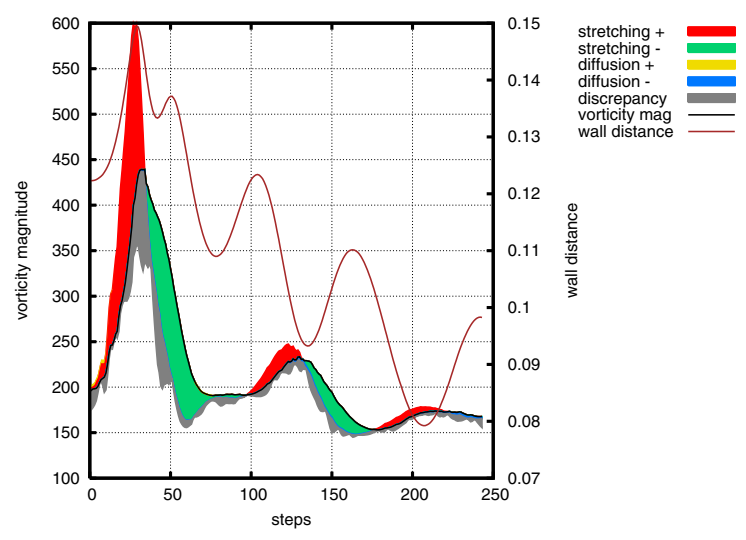

Fig. 15. Plot of pathline selected in Figure 14. 